\title{
Sulforaphane induces colorectal cancer cell proliferation through Nrf2 activation in a p53-dependent manner
}

Yunjeong Gwon ${ }^{1 \dagger}$, Jisun $\mathrm{Oh}^{2 \dagger}$ and Jong-Sang $\mathrm{Kim}^{1,2^{*}}$ (D)

\begin{abstract}
Sulforaphane is a well-known phytochemical that stimulates nuclear factor erythroid 2-related factor 2 (Nrf2)-mediated antioxidant cellular response. In this study, we found that sulforaphane promoted cell proliferation in HCT116 human colon cancer cells expressing a normal p53 gene in a dose-dependent but biphasic manner. Since p53 has been reported to contribute to cell survival by regulating various metabolic pathways to adapt to mild stress, we further examined cellular responses in both p53-wild-type (WT) and p53-knockout (KO) HCT116 cells exposed to sulforaphane in vitro and in vivo. Results demonstrated that sulforaphane treatment activated Nrf2-mediated antioxidant enzymes in both p53-WT and p53-KO cells, decreased apoptotic protein expression in WT cells but increased in KO cells in a dose-dependent manner, and increased the expression of a mitochondrial biogenesis marker PGC1a in WT cells but decreased in $\mathrm{KO}$ cells. Moreover, a low dose of sulforaphane promoted tumor growth, upregulated the Nrf2 signaling pathway, and decreased apoptotic cell death in p53-WT HCT116 xenografts compared to that in p53-KO HCT116 xenografts in BALB/C nude mice. These findings suggest that sulforaphane can influence colon cancer cell proliferation and mitochondrial function through a crosstalk between the Nrf2 signaling pathway and p53 axis.
\end{abstract}

Keywords: Sulforaphane, Colon cancer, Biphasic growth, Nrf2, p53

\section{Highlights}

- Sulforaphane increased Nrf2-mediated cytoprotective response in both p53-WT and p53-KO HCT116 cells.

- Sulforaphane promoted cell proliferation of p53-WT HCT116 cells in a biphasic manner.

- Sulforaphane attenuated apoptotic cell death and enhanced mitochondrial function in a p53-dependent manner.

- Sulforaphane promoted tumor growth, upregulated the Nrf2 signaling pathway, and decreased apoptotic cell death in p53-WT HCT116 xenografts.

*Correspondence: vision@knu.ac.kr

${ }^{\dagger}$ Yunjeong Gwon and Jisun Oh contributed equally to this work

${ }^{1}$ School of Food Science and Biotechnology, Kyungpook National

University, Daegu 41566, Republic of Korea

Full list of author information is available at the end of the article
- The findings suggest that the intake of Nrf2-activating phytochemicals could be potentially harmful to the colorectal cancer patients carrying the WT p53 gene.

\section{Introduction}

Sulforaphane (1-isothiocyanato-4-methylsulfinylbutane, SFN) is one of the most potent inducers of nuclear factor erythroid 2-related factor $2(\mathrm{Nrf2})$, a key regulator of cellular redox balance $[1,2]$. Nrf2 is sequestered by a sulfhydryl-rich cytosolic protein, kelch-like $\mathrm{ECH}$-associated protein 1 (Keap1), which facilitates the ubiquitin-proteasomal degradation of $\mathrm{Nrf} 2$ under stress-free conditions. Sulforaphane directly interacts with Keap1 and liberates Nrf2 from Keap1-dependent repression, thereby promoting nuclear translocation of Nrf2 and transcriptional upregulation of its downstream antioxidant and 
cytoprotective genes, including $H M O X 1$ encoding heme oxygenase-1 (HO-1) [3, 4]. Therefore, sulforaphane has been known to be one of the strong anti-carcinogenic phytochemicals in vitro and in vivo due to its ability to activate Nrf2-mediated cytoprotective mechanism(s) against endogenous and exogenous stressors, including reactive oxygen species (ROS) [2, 5-7].

Recently, multiple studies have indicated that Nrf2 activation in tumor cells can rather contribute to malignant transformation through metabolic reprogramming and promote tumor growth by effectively reducing ROS imposed by therapeutic exposure, and thus cause resistance to chemotherapy by protecting malignant cells from various cytotoxic stresses [8-10].

The tumor suppressor p53, called "a guardian of the genome", is known to coordinate cellular responses to diverse stress factors [11] and exert dual function as either a prooxidant ("killer") or an antioxidant ("protector") agent [12]. Depending on the cellular stress or damage, p53 can promote cell survival by activating the repair system, or it can cause cell death by inducing apoptosis. Similarly, tumor cell viability and death can be modulated by the action of p53 in the context of tumor growth. Indeed, p53 is often mutated in cancer cells, which leads to perturbation of cellular mechanisms regulating cell survival and growth [13].

Intriguingly, Nrf2-mediated antioxidant response is associated with the function of p53 [14]. It has been reported that p53 directly binds to the Nrf2-activated promoter elements and thus represses the expression of antioxidant genes. In addition, the p53 downstream effector, p21, binds to Keap1 and stabilizes Nrf2. An Nrf2 downstream enzyme, $\mathrm{NAD}(\mathrm{P}) \mathrm{H}$ :quinone oxidoreductase (NQO1), interacts with p53 and blocks the proteasomal degradation of $\mathrm{p} 53$.

However, little is known about the interrelationship between Nrf2 and p53 in colorectal cancer cells that are responsive to sulforaphane. In the present study, we reported a possible detrimental effect of sulforaphane via its Nrf2-activating capability on colorectal cancer in accordance with the presence of p53 in vitro and in vivo. The findings of this study would provide insights into the possibility that indiscreet consumption of strong Nrf2 activators may cause harm to colorectal cancer patients, as opposed to the anticipated therapeutic potential.

\section{Materials and Methods}

\section{Cell culture}

The two human colorectal cancer cell lines, HCT116 (p53-wild-type; p53-WT; p53 ${ }^{+/+}$; Korean Cell Line Bank, Seoul, South Korea) and its mutant (p53-knockout; p53-KO; p53-/-; a generous gift from Prof. Young Ho Kim, Kyungpook National University, Daegu, South
Korea, which was originally provided by Dr. Bert Vogelstein, Johns Hopkins University, Baltimore, MD, USA) were cultivated in Dulbecco's modified Eagle's medium (DMEM) containing 10\% fetal bovine serum (FBS), $25 \mathrm{mM}$ hydroxyethyl piperazineethanesulfonic acid (HEPES), 1\% minimum essential medium with nonessential amino acids (NEAA), and $1 \%$ penicillin-streptomycin (all from Invitrogen/Life Technologies, Carlsbad, CA, USA). For subculture, cells were rinsed in phosphate-buffered saline (PBS; Life Technologies), detached using 0.05\% Trypsin-EDTA (Welgene Inc., Gyeongsan, South Korea), harvested, and plated in culture dishes (Thermo Fisher Scientific, Waltham, MA, USA). Cells were maintained in a culture incubator (Sanyo, Osaka, Japan; $37{ }^{\circ} \mathrm{C}, 5 \% \mathrm{CO}_{2} / 95 \%$ air, humidified atmosphere).

\section{Cell viability}

Cell viability was examined using Cell Counting Kit-8 (CCK-8; Dojindo Laboratories, Kumamoto, Japan) as previously described [15]. Cells were seeded at a density of $5 \times 10^{3}$ cells per well in a 96-well plate. After $24 \mathrm{~h}$, cells were treated with various concentrations of sulforaphane (Enzo Life Sciences Inc., Farmingdale, NY, USA) in the absence or presence of an HO-1 inhibitor (tin protoporphyrin IX, SnPP; Cayman Chemical, Ann Arbor, MI, USA), an Nrf2 signaling inhibitor (brusatol; Carbosynth Ltd., Newbury, Berkshire, UK), or a mitochondrial complex I inhibitor (rotenone; Sigma-Aldrich, St. Louis, MO, USA) for $24 \mathrm{~h}$. The absorbance reflecting the number of living cells in each well was measured at $450 \mathrm{~nm}$ using a microplate reader (Sunrise ${ }^{\mathrm{TM}}$, Tecan Group Ltd., Männedorf, Switzerland).

\section{Western blot analysis}

Nuclear and cytoplasmic proteins were separately fractionated from the harvested cells using NE-PER ${ }^{\circledR}$ Nuclear and Cytoplasmic Extraction Reagents (Thermo Fisher Scientific) as previously described [15]. The primary antibodies used were rabbit anti-Nrf2 (abcam, Cambridge, UK), rabbit anti-HO-1 (abcam), rabbit anti-LC3 (NOVUS Biologicals, Littleton, CO, USA), mouse anti-cytochrome $\mathrm{C}$, mouse anti-Bcl-2, mouse anti-Bax, mouse anti-Lamin B1, and mouse anti- $\beta$-actin (Santa Cruz Biotechnology, Dallas, TX, USA). The secondary antibodies used were anti-rabbit or anti-mouse immunoglobulin G, conjugated to horseradish peroxidase (Santa Cruz Biotechnology).

\section{Quantitative polymerase chain reaction (qPCR) analysis}

Total DNA was extracted from the harvested cells using a column-based isolation kit (DNeasy Mini Kit; Qiagen, Hilden, Germany) according to the manufacturer's instructions. The extracted DNA was quantified based on the absorbance at $280 \mathrm{~nm}$ using BioSpectrometer ${ }^{\circledR}$ 
basic (Eppendorf, Hamburg, Germany). SYBR Greenbased real-time PCR was performed using LightCycler ${ }^{\circledR}$ Multiplex Masters (Roche, Basel, Switzerland) with the primer sets (Bioneer Corp., Daejeon, South Korea; Additional File 1: Table S1) on LightCycler ${ }^{\circledR}$ Nano Instrument (Roche). The mitochondrial DNA level was normalized to the nuclear DNA level.

\section{Animal experiments}

All animal experiment was conducted under the approval of the Institutional Animal Care and Use Committees at Kyungpook National University (Approval Number 2017-0038), and performed in accordance with relevant guidelines and regulations. Six-week-old male BALB/c nude mice (Orient Bio Inc., Seongnam, South Korea) weighing $18 \pm 2 \mathrm{~g}$ were acclimated for a week under standard conditions (temperature, $22 \pm 2{ }^{\circ} \mathrm{C}$; humidity, $45 \pm 5 \%$; illumination, 150-300 lux) with free access to drinking water and standard mouse chow pellets (Daehan Bio Link, Eumseong, South Korea).

Each 7-week-old mouse was subcutaneously injected with both types of HCT116 cells, p53-WT and p53-KO, on its left and right flanks, respectively. Cells $\left(2.5 \times 10^{6}\right.$ per injection) were resuspended in $100 \mu \mathrm{L}$ of a $1: 1$ mixture of cold PBS and Matrige ${ }^{\circledR}$ Matrix (Corning Inc., Corning, NY, USA) and transplanted (set at day 0). A week after transplantation (at day 7), mice were randomly divided into four groups (5-6 mice per group; Table 1 ): no treatment (vehicle only; negative control) or sulforaphane treatment at three different doses [low, $2.5 \mathrm{mg}$ / $\mathrm{kg}$ body weight (BW); medium, $10 \mathrm{mg} / \mathrm{kg} \mathrm{BW;} \mathrm{high,}$ $25 \mathrm{mg} / \mathrm{kg} \mathrm{BW]}$. Sulforaphane was prepared in vehicle, composed of $10 \% \mathrm{v} / \mathrm{v}$ dimethyl sulfoxide (DMSO) and $10 \% \mathrm{v} / \mathrm{v}$ Tween-80 in sterilized saline, and was intraperitoneally administered on days 7 and 9. Body weight and xenograft volumes were monitored every other day for two weeks. The length and width of each xenograft were

Table 1 Experimental groups of BALB/c nude mice harboring HCT116 xenografts

\begin{tabular}{lll}
\hline Group & $\begin{array}{l}\text { Number of animals } \\
\text { (n) }\end{array}$ & $\begin{array}{l}\text { Dose } \\
\text { (mg/kg BW) }\end{array}$ \\
\hline Control (vehicle only) $^{\text {a }}$ & 6 & 0 \\
SFN_Low $^{\text {b }}$ & 5 & 2.5 \\
SFN_Medium $^{c}$ & 5 & 10 \\
SFN_High $^{d}$ & 5 & 25
\end{tabular}

a Vehicle contains 10\% DMSO, 10\% v/v Tween-80, and $80 \%$ sterilized saline.

b SFN_Low, experimental group treated with a low dose $(2.5 \mathrm{mg} / \mathrm{kg}$ BW) of sulforaphane

c SFN_Medium, experimental group treated with a medium dose $(10 \mathrm{mg} / \mathrm{kg}$ BW) of sulforaphane

d SFN_High, experimental group treated with a high dose (25 mg/kg BW) of sulforaphane measured using a caliper (Mitutoyo Corp., Kanagawa, Japan). After sacrificing the mice at day 21, the xenografts were dissected, weighed, and frozen at $-80{ }^{\circ} \mathrm{C}$ before subsequent analyses.

\section{Immunohistochemistry}

Dissected xenograft tumor tissues were fixed in 3.7\% $\mathrm{v} / \mathrm{v}$ formaldehyde in PBS, paraffin-embedded, and sectioned [16]. The sections were placed on microscope slides, air-dried at $37{ }^{\circ} \mathrm{C}$ for $12 \mathrm{~h}$, and stored at $4{ }^{\circ} \mathrm{C}$ before being processed for immunostaining. The prepared sections were deparaffinized and immunostained using a FITC-conjugated antibody against proliferation cell nuclear antigen (PCNA, a proliferation cell marker; Santa Cruz Biotechnology). Nuclei were counterstained with $1 \mu \mathrm{g} / \mathrm{mL}$ of 4',6-diamidino-2-phenylindole (DAPI). After mounting, the slides were observed under a fluorescence microscope (Eclipse 80i, Nikon, Tokyo, Japan).

\section{Terminal deoxynucleotidyl tranferase (TdT) dUTP nick-end labeling (TUNEL) assay}

The deparaffinized sections were subjected to staining using the ApopTag ${ }^{\circledR}$ Red In Situ Apoptosis Detection Kit (Millipore, Burlington, MA, USA) according to the manufacturer's instructions. Briefly, the sections were equilibrated in digoxigenin nucleotide-containing reaction buffer and then allowed to react with $\mathrm{TdT}$. The digoxigenin-conjugated DNA fragments were observed under a fluorescence microscope by tightly binding with anti-digoxigenin conjufated to rhodamine.

\section{Statistical analysis}

The statistical significance of data was determined by one-way analysis of variance (ANOVA) followed by a post-hoc Duncan's multiple-range test, using SPSS software (SPSS Inc., Chicago, IL, USA). The $p$-value less than 0.05 was considered to be statistically significant. Statistical differences were indicated using asterisks or different alphabetical letters.

\section{Results}

Sulforaphane triggered biphasic survival response in p53-expressing colorectal cancer cells

To examine the effect of sulforaphane on the growth of human colon cancer cells and its dependency on the expression of p53, p53-WT and p53-KO HCT116 cells were cultured in the presence of various concentrations of sulforaphane $(0,0.5,1,5,10,20$, and $50 \mu \mathrm{M})$. After $24 \mathrm{~h}$ of incubation, cell viability was assessed. Low doses of sulforaphane $(\leq 5 \mu \mathrm{M})$ were non-cytotoxic to both types of HCT116 cells and significantly increased cell proliferation in p53-WT HCT116 cells but not in p53-KO HCT116 cells (Fig. 1a; Additional File 2: Fig. S1A). 

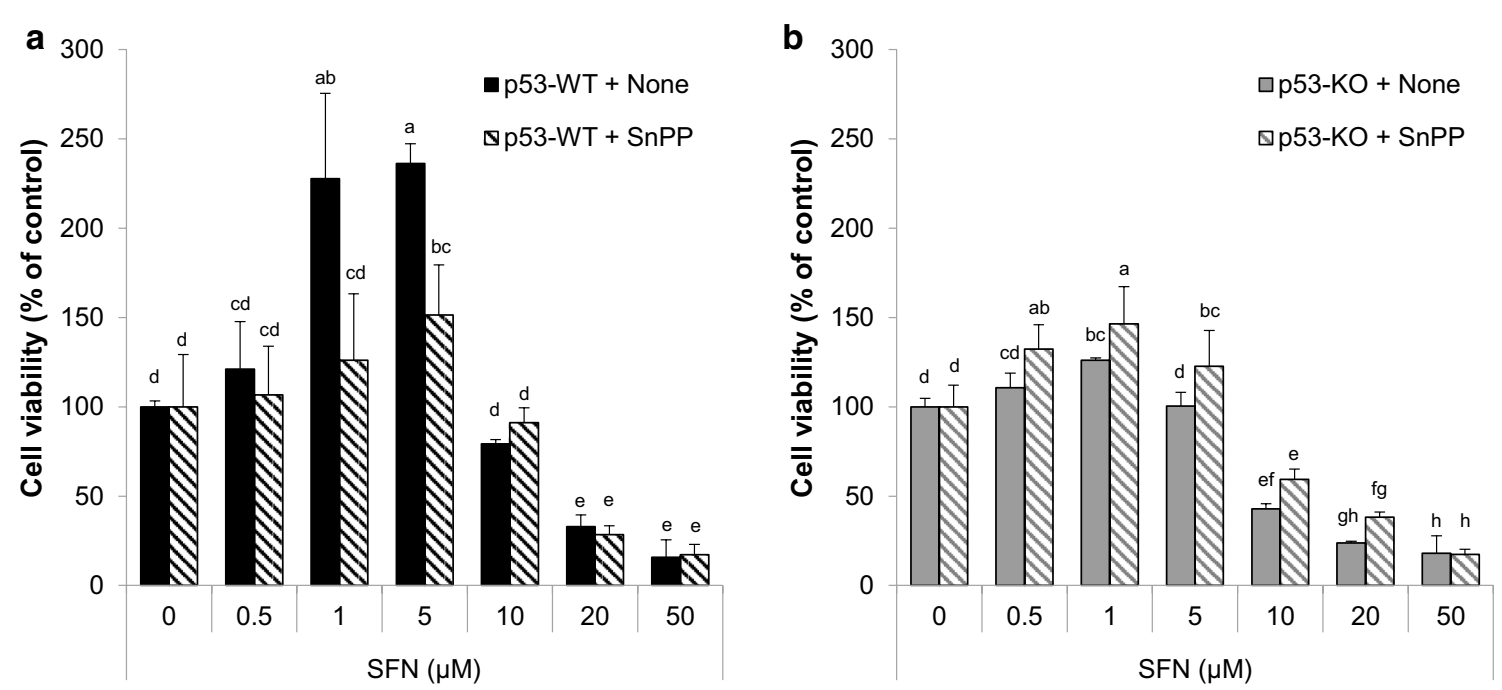

Fig. 1 Sulforaphane-induced p53-WT HCT116 cell growth was attenuated by an HO-1 inhibitor, SnPP. Both types of HCT116 cells, p53-WT (a) and p53-KO (b), were cultured with various concentrations of sulforaphane $(0,0.5,1,5,10,20$, and $50 \mu M)$ in the absence or presence of SnPP (10 $\mu M)$. After $24 \mathrm{~h}$ of incubation, cell viability was assessed by CCK-8 assay. Values represent mean \pm standard error of mean (SEM) from three independent experimental sessions $(\mathrm{N}=3)$. Statistical differences are indicated by different alphabetical letters for multiple comparisons $(p<0.05)$

However, sulforaphane at concentrations of $\geq 10 \mu \mathrm{M}$ caused cell death and its effect was more pronounced in p53-KO cells than in p53-WT cells. These results demonstrated that sulforaphane promoted HCT116 colon cancer cell proliferation in a biphasic manner, which was more prominent in p53-expressing cells than in p53-deficient cells.

\section{Sulforaphane-induced biphasic growth of p53-WT HCT116} cells was mediated through Nrf2 signaling pathway

To determine whether sulforaphane-induced cell proliferation is attributable to Nrf2-mediated signaling pathway, p53-WT and p53-KO HCT116 cells were treated with sulforaphane in combination with either the HO-1 inhibitor SnPP (10 $\mu$ M; Fig. 1b) or the Nrf2 inhibitor brusatol (0.5 nM; Additional File 2: Fig. S1B). Cell viability assay showed that sulforaphane-induced cell growth was significantly reduced in p53-WT HCT116 cell cultures following treatment with SnPP or brusatol, while the growth of p53-KO cells was not influenced by inhibitor treatment. These results suggest that sulforaphane accelerated the growth of p53-expressing HCT116 cells via the Nrf2 signaling pathway.

Although sulforaphane at low doses enhanced HCT116 cell proliferation probably through activation of the Nrf2 signaling pathway, the protein levels of nuclear Nrf2 and cytoplasmic HO-1 were increased in both types of cells in a dose-dependent manner, regardless of p53 status (Fig. 2). These findings implicate that sulforaphaneinduced cell growth is presumably regulated by the combinatorial effect of Nrf2-mediated cellular defense signal and p53-associated cell survival signal.

\section{Sulforaphane decreased apoptotic cell death in p53-WT cells, but not in p53-KO cells}

Sulforaphane-treated cells were further examined for the expression of apoptosis-associated proteins by western blot analysis (Fig. 3). The ratio of anti-apoptotic protein, $\mathrm{Bcl}-2$, to pro-apoptotic protein, Bax, was significantly increased in p53-WT cells, but not in p53-KO cells following sulforaphane treatment. This suggests that the inhibitory activity of sulforaphane against apoptotic cell death may require the function of $\mathrm{p} 53$. In addition, considering that HCT116 cells harboring functional p53 expressed a high ratio of LC3-II to LC3-I (Additional File 2: Fig. S2), sulforaphane-induced cell growth is likely attributable to upregulation of autophagy in the presence of p53.

\section{A low dose of sulforaphane promoted the growth of p53-WT HCT116 xenografts}

To examine the effect of sulforaphane treatment on tumor growth in vivo, p53-WT and p53-KO HCT116 cells were xenografted into the left and right sides of the posterior flank of the same BALB/c nude mouse, respectively, by subcutaneous injection. The mice were administered with three different doses of sulforaphane by intraperitoneal injection (Fig. 4a; Table 1). Body weight and tumor volume for each group were regularly monitored. The 

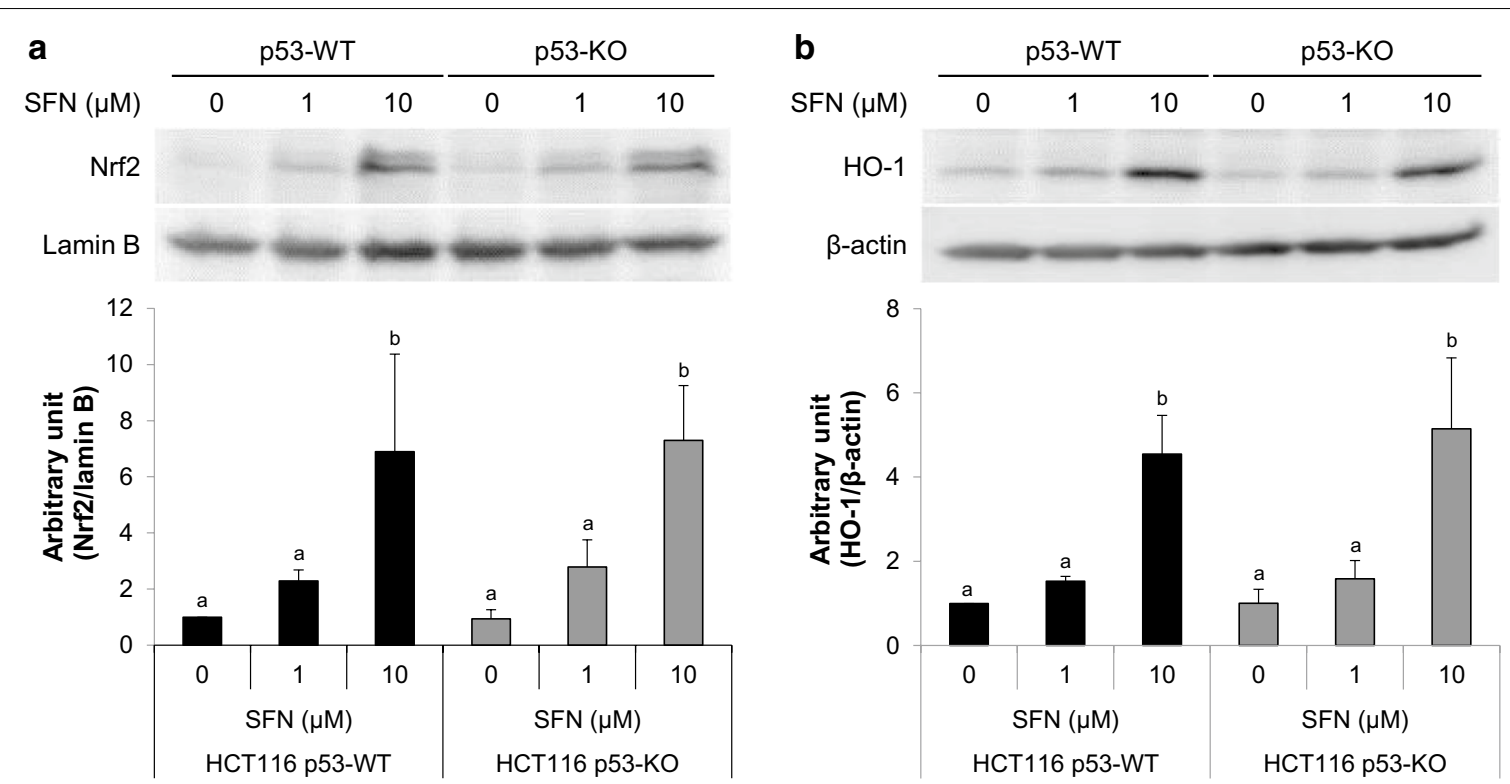

Fig. 2 Sulforaphane increased nuclear Nrf2 and cytoplasmic HO-1 levels in both p53-WT and p53-KO cells. HCT116 cells were treated with sulforaphane for $8 \mathrm{~h}$. The nuclear and cytoplasmic fractions were prepared from the harvested cells. The protein levels of nuclear Nrf2 (a) and cytoplasmic HO-1 (b) were examined in both p53-WT and p53-KO cells by western blot analysis. Upper, a representative western blot image; lower, quantified data. Values represent mean \pm SEM ( $N=3$ for $\mathbf{a} ; \mathrm{N}=6$ for $\mathbf{b}$ ). Statistical differences are indicated by different alphabetical letters for multiple comparisons $(p<0.05)$

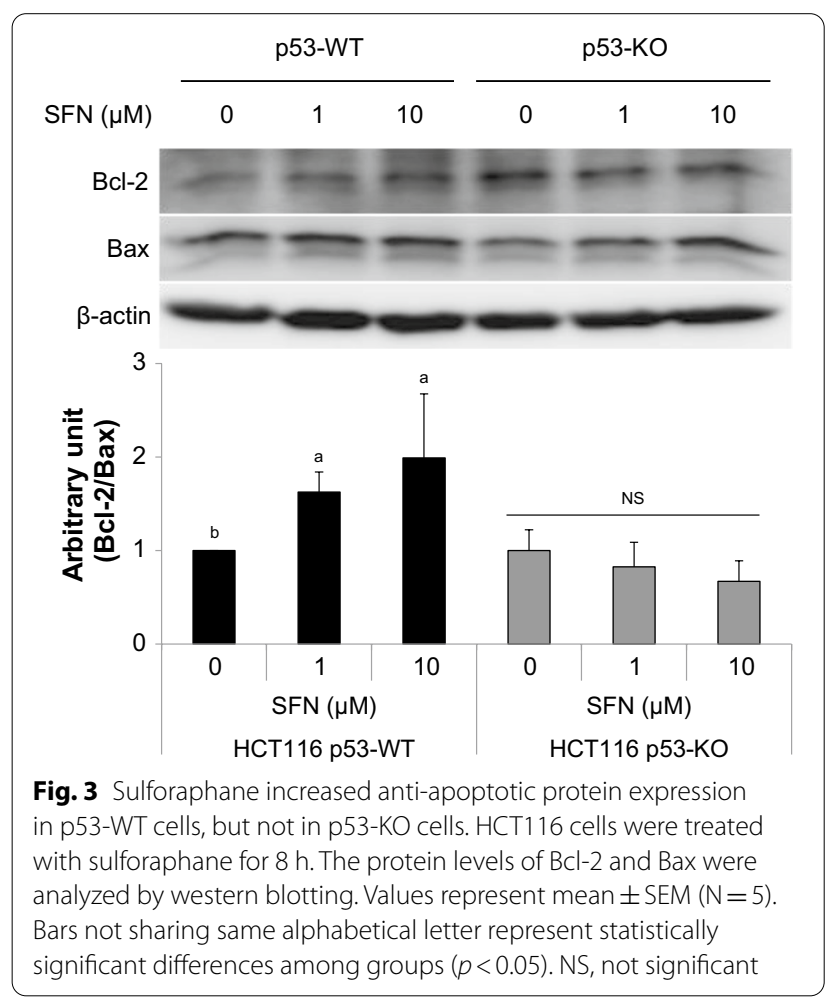

treatment had no significant effect on the average body weight among the groups (Fig. $4 \mathrm{~b}$ ). The tumor volumes were gradually increased but not significantly different between the p53-WT and p53-KO HCT116 xenografts in the control group (Fig. 4c). However, the tumor volume in mice transplanted with p53-WT HCT116 cells was significantly increased following treatment with low-dose sulforaphane, while medium- or high-dose sulforaphane treatment had little effect (Fig. $4 \mathrm{~d}$ and f). The tumor volume in mice transplanted with p53-KO cells was not significantly affected by sulforaphane treatment (Fig. $4 \mathrm{e}$ and g).

\section{Sulforaphane activated the Nrf2 signaling pathway in p53-WT xenografts but promoted apoptosis in p53-KO xenografts}

To address the possibility that the cytoprotective effect exerted by Nrf2-mediated antioxidative machinery contributes to tumor growth [8], the expression levels of nuclear Nrf2 and cytoplasmic HO-1 in the xenografts were examined (Fig. 5a, b). Sulforaphane treatment appeared to increase the levels of those proteins only in p53-WT HCT116 xenografts but not in p53-KO xenografts, although the differences were not statistically significant.

When examined for the expression of apoptosis-associated proteins, such as Bcl-2, Bax, and cytochrome $\mathrm{C}$ in $\mathrm{p} 53-\mathrm{WT}$ or $\mathrm{p} 53-\mathrm{KO}$ xenografts 


\section{(See figure on next page.)}

Fig. 4 Sulforaphane promoted tumor growth only in the presence of p53. a Animal experimental scheme; the two types of HCT116 cells were subcutaneously injected on day 0 and grown for 7 days. Xenografted tumor-bearing mice were intraperitoneally administered with vehicle (negative control; Control), low-dose sulforaphane (SFN_Low), medium-dose sulforaphane (SFN_Med), or high-dose sulforaphane (SFN_High) on days 7 and 9 . The body weight and tumor volume of each mouse were monitored every other day. $\mathbf{b}$ No significant effect of sulforaphane on the body weight of BALB/c nude mice bearing HCT116 cell xenografts was observed. Values represent mean \pm standard deviation (SD) $(n=5$ or 6$)$. $\mathbf{c}$ The xenografts formed in the mice gradually grew in size but were not significantly different from those formed in control (no treatment) mice. $\mathbf{d}$, e The volumes of p53-WT (d) or p53-KO (e) xenografts were increased with sulforaphane treatment. WT xenografts were significantly increased in size by treatment with a low dose of sulforaphane, while KO xenografts were not affected by sulforaphane treatment. Values represent mean \pm SD $(n=5$ or 6). Asterisk indicates significant differences among the conditions. $\mathbf{f}, \mathbf{g}$ The picture of p53-WT (f) or p53-KO (g) xenograft tumors dissected from the mice after sacrifice

dissected from sulforaphane-treated mice (Fig. 5c, d), no significant differences were observed in the Bcl-2 to Bax ratio and cytochrome $\mathrm{C}$ expression in $\mathrm{p} 53-\mathrm{WT}$ xenografts regardless of sulforaphane treatment. However, the relative expression of Bax and cytochrome $\mathrm{C}$ was significantly increased in p53-KO xenografts treated with medium- or high-dose sulforaphane. These results were supported by those of immunohistochemical analysis of tumor xenograft sections using TUNEL assay and a proliferating cell marker, PCNA (Fig. 5e, f). Compared to the control (no sulforaphane treatment), more number of TUNEL-stained cells was observed in xenografts treated with high-dose sulforaphane, regardless of p53 status. However, p53-WT xenografts contained remarkably more number of cells expressing PCNA than $\mathrm{p} 53-\mathrm{KO}$ xenografts following low-dose sulforaphane treatment. These results demonstrated that low-dose sulforaphane promoted cell proliferation in p53-WT xenografts, but caused apoptotic cell death in p53-KO xenografts in which Nrf2 and $\mathrm{HO}-1$ expression levels were relatively lower than those in p53-WT xenografts.

\section{Sulforaphane facilitated mitochondrial function in p53-dependent HCT116 cell growth}

To further elucidate a possible correlation between HCT116 cell fate and mitochondrial function, cells were treated with sulforaphane for $8 \mathrm{~h}$ and then examined for mitochondrial DNA abundance and protein expression of $\mathrm{PGC} 1 \alpha$, a mitochondrial biogenesis marker (Fig. 6). We found that sulforaphane treatment remarkably decreased mitochondrial DNA to nuclear DNA ratio (mtDNA/nDNA) and PGC1 $\alpha$ protein level only in p53-KO cells. These results demonstrated that p53 contributed to the maintenance of mitochondrial DNA copy number and stimulation of biogenesis in sulforaphane-treated HCT116 cells. Interestingly, sulforaphane treatment increased mitochondrial respiratory capacity in p35-WT cells, but not in p53-KO cells (Additional File 2: Fig. S3). These findings suggest that sulforaphane promoted mitochondrial biogenesis and cellular respiration in a p53-dependent mode.

\section{Discussion}

Recent studies have suggested that Nrf2-mediated antioxidant and detoxifying enzyme induction contributes to the resistance of cancer cells to therapeutic agentinduced ROS insults, the arsenal for cancer treatment $[8$, 17-21]. Sulforaphane is a well-known Nrf2 activator [22-24]. Our present study demonstrated that sulforaphane, at relatively low doses, can induce biphasic growth of HCT116 human colorectal cancer cells that are, in particular, expressing p53. Hence, in an effort to elucidate the interrelationship between Nrf2 and p53 in colorectal cancer cell proliferation, we examined cellular responses in both p53-WT and p53-KO HCT116 cells exposed to sulforaphane in vitro and in vivo. Our results showed that sulforaphane-induced tumor growth was accompanied by Nrf2-mediated induction of antioxidant enzymes, upregulated expression of anti-apoptotic proteins, and increased mitochondrial biogenesis and respiration, intriguingly only in the cells harboring the active p53 gene (Fig. 7). In addition, p53-deficient cells seemed to easily undergo apoptotic cell death in response to sulforaphane, compared to $\mathrm{p} 53$-expressing cells.

Sulforaphane treatment, as expected, activated Nrf2 in HCT116 cells in a dose-dependent manner regardless of the presence of p53. Moreover, sulforaphane-treated 
a
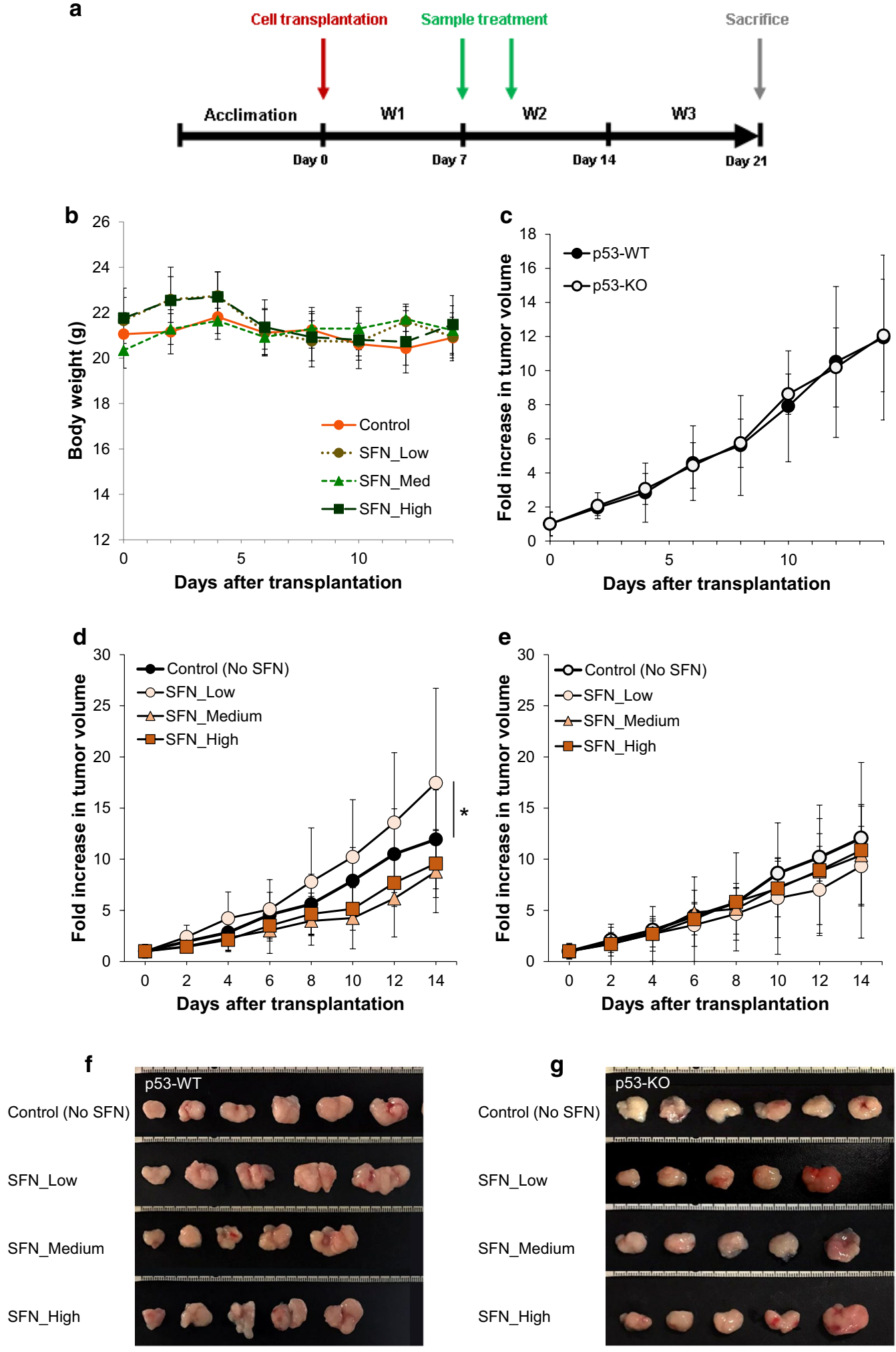

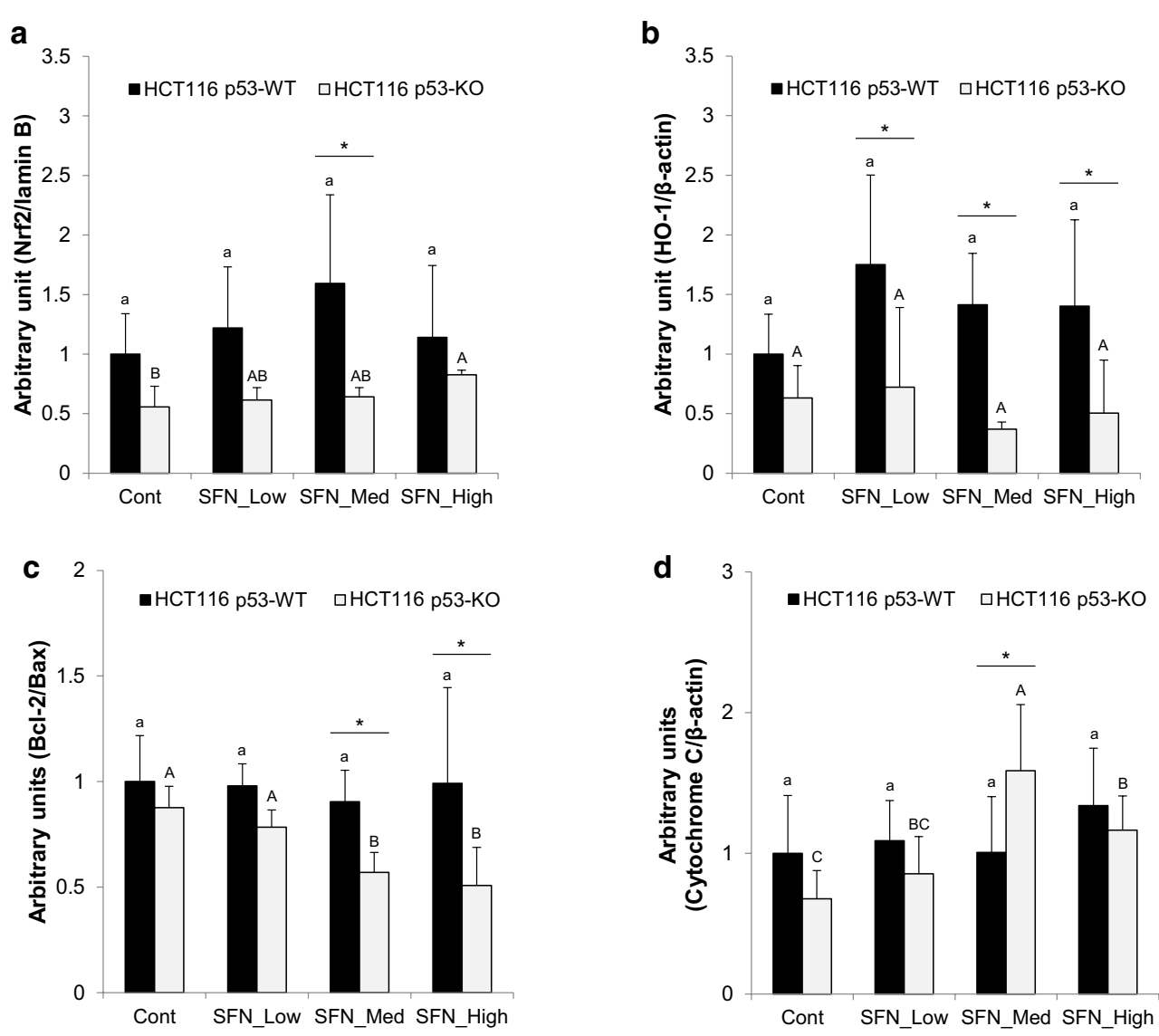

e

p53-WT

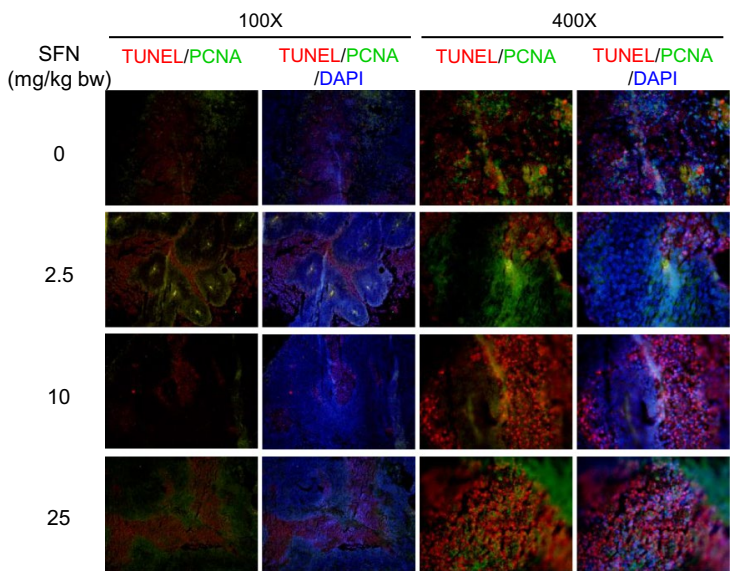

$\mathbf{f}$

p53-KO

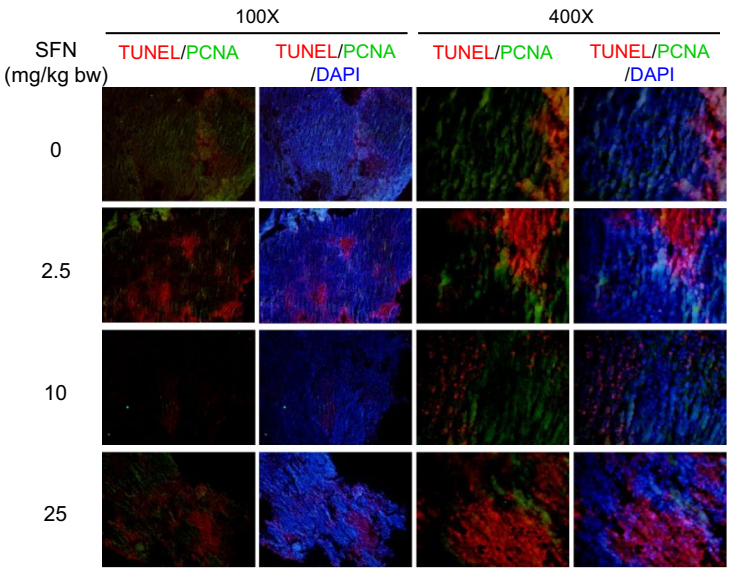

Fig. 5 Sulforaphane induced cytoprotective response in p53-positiveWT xenografts. The nuclear and cytoplasmic proteins were fractionated from the dissected xenograft tissues. $\mathbf{a}$, b Sulforaphane treatment tended to increase the protein levels of Nrf2 (a) and HO-1 (b) in p53-WT xenografts compared to those in $\mathrm{p} 53-\mathrm{KO}$ xenografts. $\mathbf{c}$, $\mathbf{d}$ Sulforaphane treatment increased the expression of apoptotic markers, a ratio of $\mathrm{BCl}-2$ to $\mathrm{Bax}$ (c) and cytochrome C (d), in p53-KO xenografts but not in p53-WT xenografts. Values represent mean \pm SD $(n=5$ or 6$)$. Different lower and upper cases indicate statistically significant differences among p53-WT and p53-KO xenografts, respectively. Asterisks indicate statistically significant differences between WT and KO xenografts within the same treatment group. e, f Sectioned tumor slices from p53-WT (e) or p53-KO xenografts (f) were subjected to immuno-staining with a FITC-conjugated anti-PCNA (green fluorescence) and TUNEL staining with a rhodamine-conjugated anti-digoxigenin (red fluorescence). Representative images were obtained at 100X or 400X magnifications. Sulforaphane-treated p53-WT xenografts exhibited a higher ratio of PCNA-positive cells over TUNEL-stained cells than p53-KO xenografts 

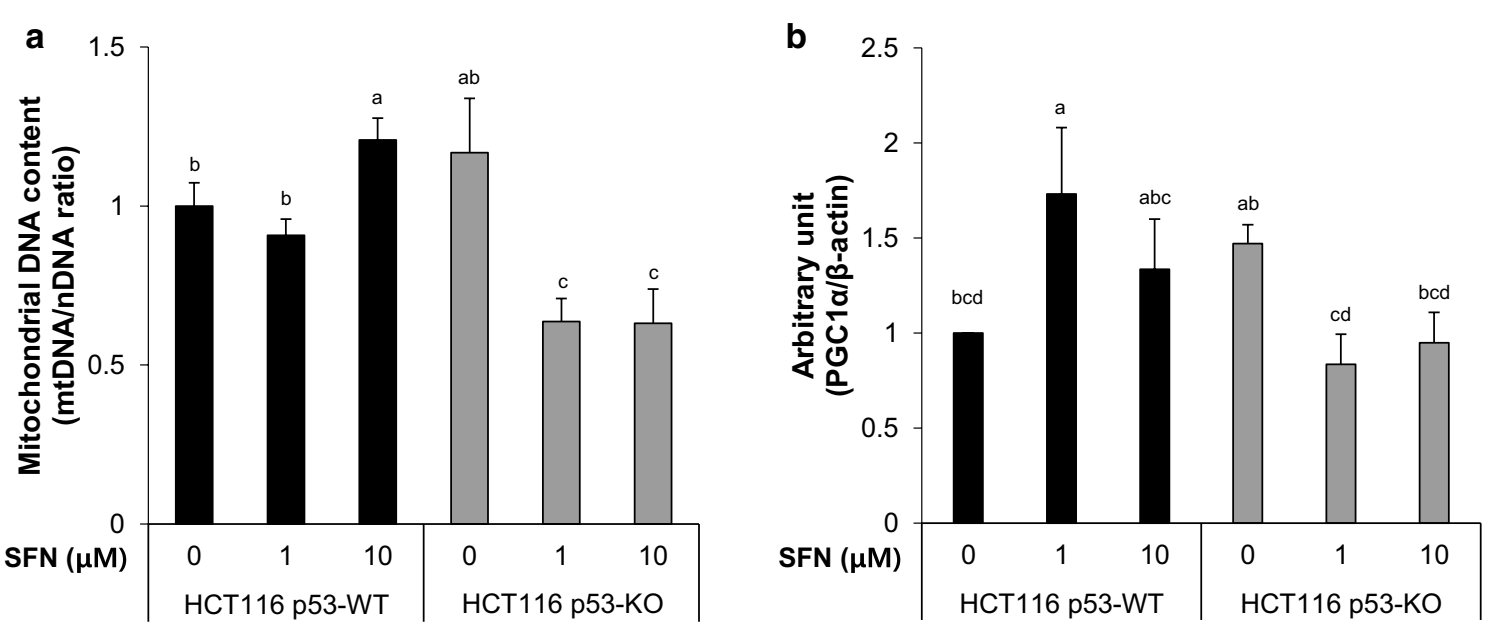

Fig. 6 Sulforaphane impeded mitochondrial biogenesis in the absence of p53. Both types of HCT116 cells were treated with sulforaphane $(0,1$, or $10 \mu \mathrm{M}$ ) for $8 \mathrm{~h}$. $\mathbf{a}$ The ratio of mitochondrial DNA (mtDNA) to nuclear DNA (nDNA) in the HCT116 cells was quantified using real-time qPCR. $\mathbf{b}$ PGC1 a protein expression was examined using western blotting and densitometric analysis. Values represent mean \pm SEM $(N=3)$. Statistical differences are indicated by different alphabetical letters for multiple comparisons

\section{HCT116 cell cultures or mouse xenografts}
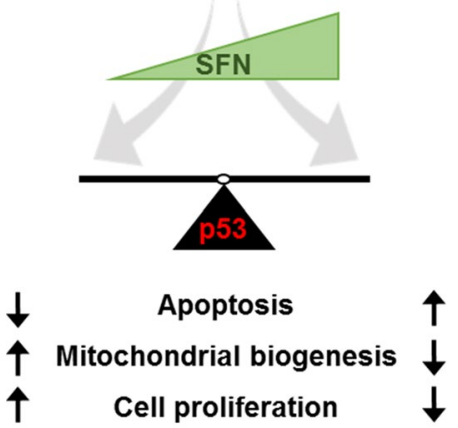

\section{Cell growth}

\section{Cell death}

Fig. 7 An illustrative summary of the observations in this study

HCT116 cells displayed hormetic growth; that is, cell growth was stimulated at sulforaphane concentrations of $\leq 5 \mu \mathrm{M}$ but suppressed at concentrations of $\geq 10 \mu \mathrm{M}$, and this effect was prominent in the presence of p53. This biphasic growth was attenuated by treatment with an antagonist of either Nrf2 or HO-1, suggesting that sulforaphane-induced HCT116 cell propagation is mediated via the Nrf2 signaling pathway exclusively in p53-existing circumstance.

p53-associated biphasic growth of sulforaphanetreated HCT116 cells was also observed in the xenograft mouse model. A low dose of sulforaphane $(2.5 \mathrm{mg} / \mathrm{kg}$ $\mathrm{BW}$ ) increased tumor volume in mice transplanted with
p53-WT HCT116 cells, with concomitant activation of Nrf2 and its downstream enzymes. This was consistent with the in vitro observation. Interestingly, both p53-WT and p53-KO xenografts highly expressed apoptosisassociated proteins when exposed to a high dose of sulforaphane. Furthermore, p53-WT xenografts contained more number of proliferating cells than $\mathrm{p} 53-\mathrm{KO}$ xenografts following low-dose sulforaphane treatment. This indicates that sulforaphane-induced biphasic growth of p53-expressing HCT116 cells in vitro and in vivo is governed by summation of Nrf2-mediated cytoprotective response and differential p53-mediated cell signaling, depending on the dose of sulforaphane.

It has been reported that p53, a tumor suppressor, restricts Nrf2-dependent upregulation of antioxidant enzymes by directly binding to their promoter elements $[14,25]$. Conversely, p53 can promote cell survival in a p21-dependent manner: $\mathrm{p} 21$, a downstream target gene of p53, stabilizes Nrf2 by enhancing Keap1 disruption through p62-mediated autophagic degradation, thereby causing upregulation of Nrf2 downstream genes [14, 26, 27]. In response to certain factors stimulating intracellular ROS generation, p53 upregulates the expression of antioxidant genes, protecting cells from ROS-induced insults under low levels of ROS, while under high levels of ROS, p53 is highly expressed and activates prooxidant genes, resulting in apoptosis [28]. This suggests that $\mathrm{p} 53$ plays a crucial role in cell fate decision-making [28-30], that is, p53 coordinates intracellular mechanisms either to endure by activating Nrf2-mediated antioxidant response or to perish by triggering the prooxidant process and apoptosis upon 
oxidative insults. In this regard, sulforaphane-stimulated p53-WT HCT116 colorectal cancer cell growth at the relatively low doses in vitro and in vivo is most likely attributable to enhanced Nrf2-mediated cell survival and inhibited apoptotic cell death.

Following treatment with sulforaphane, p53-WT HCT116 cells displayed a higher ratio of LC3-II to LC3-I and expressed abundant mtDNA and PGC1 $\alpha$ protein compared to $\mathrm{p} 53-\mathrm{KO}$ cells, indicating that p53-deficient cells underwent less active autophagy and mitochondrial biogenesis than p53-expressing cells. This suggests that sulforaphane-induced HCT116 cell proliferation is associated with mitochondrial function under the control of p53.

p53 is well known to modulate autophagy through the orchestrated work of its downstream genes, such as TP53-induced glycolysis and apoptosis regulator (TIGAR) or damage-regulated autophagy modulator (DRAM) [31, 32]. Considering that dysregulation of autophagy leads to accumulation of damaged proteins, increased oxidative stress, and dysfunctional mitochondria [33], the upregulation of autophagy in the presence of p53 is likely to be advantageous for cancer cell survival.

Our quantitative PCR results demonstrated that mitochondrial abundance assessed by mitochondrial DNA content relative to nuclear DNA content was remarkably altered by sulforaphane treatment in p53-KO cells, compared to that in p53-WT cells. This implies that p53 contributes to mitochondrial dynamics [34] and structural homeostasis $[35,36]$ in response to an exogenous Nrf2 activator. Moreover, Nrf2 activation can stimulate the expression of proteins involved in mitochondrial biogenesis $[37,38]$ and attenuate oxidative stress-induced mitochondrial decay [39]. These findings suggest that mitochondrial function is regulated by the Nrf2 signaling pathway [40-43] in the presence of functional p53. However, a detailed understanding of the cross-talk mechanisms between mitochondrial biogenesis and colorectal cancer cell proliferation requires further study.

The present study demonstrated that sulforaphane induced HCT116 cell proliferation in vitro and in vivo through the Nrf2 signaling pathway in a biphasic and p53-dependent manner. HCT116 cell growth is associated with increased Nrf2-mediated cytoprotective response, attenuated apoptotic cell death, and enhanced mitochondrial function in the presence of functional p53. These observations suggest that the intake of Nrf2-activating phytochemicals by colorectal cancer patients carrying the WT p53 gene, would be potentially harmful.

Sulforaphane, a typical Nrf2 activator, is commonly generated from glucoraphanin by the enzyme myrosinase. The content of sulforaphane in broccoli and its sprout is ranged from 6 to $1555.95 \mu \mathrm{g} / \mathrm{g}$ dry weight, depending on varieties and parts $[44,45]$. As the dose of $2.5 \mathrm{mg} / \mathrm{kg}$ BW sulforaphane that increased tumor growth in mice can be converted to $14 \mathrm{mg}$ for a human weighting $70 \mathrm{~kg}$ [46], the intake of about $10 \mathrm{~g}$ of dried broccoli sprout could increase tumor proliferation in the worst case. However, it is least likely that a normal diet with vegetables has any significantly harmful effect on cancer patients as most cruciferous vegetables, including red cabbage, radish, and cabbage, reportedly contain sulforaphane $200 \mu \mathrm{g} / \mathrm{g}$ dry weight or lower [47]. Nonetheless, it is recommended that cancer patients avoid excessive intake of cruciferous vegetables with high levels of sulforaphane or its precursor glucoraphanin.

\section{Supplementary Information}

The online version contains supplementary material available at https://doi. org/10.1186/s13765-020-00578-y.



Additional file1: Table S1. Primer sets for quantitative real-time PCR.

Additional file2: Fig S1. Sulforaphane-induced p53-WT HCT116 cell growth was attenuated by an Nrf2 inhibitor, brusatol. HCT116 cells, p53-WT (A) and p53-KO (B), were treated with sulforaphane in the absence or presence of brusatol $(0.5 \mathrm{nM})$ for $24 \mathrm{~h}$, followed by cell viability assay. Values represent mean \pm SEM $(\mathrm{N}=3)$. Statistical differences are indicated by different alphabetical letters for multiple comparisons. Fig S2. Sulforaphane-induced biphasic growth of p53-WT HCT116 cells was presumably attributed to upregulated autophagy. HCT116 cells were treated with sulforaphane for $8 \mathrm{~h}$. The cytoplasmic fraction was prepared from the cell lysate. Protein levels of LC3-II and LC3-I were determined by western blot analysis. Values represent mean \pm SEM $(N=3)$. Statistical differences were indicated by different alphabetical letters for multiple comparisons. Fig S3. Sulforaphane increased mitochondrial respiration in p53-WT HCT116 cells. p53-WT (A) and p53-KO (B) HCT116 cells were treated with 0,1 , or $10 \mu \mathrm{M}$ of sulforaphane for $8 \mathrm{~h}$. Oxygen consumption rate $(\mathrm{OCR})$ was measured by the Seahorse Extracellular Flux Analyzer. Oligomycin, FCCP, and Rotenone/Antimycin A were sequentially injected at the indicated time points.

\section{Acknowledgements}

This work was supported by the National Research Foundation of Korea (NRF) grant funded by the Ministry of Science and ICT (MSIT), Republic of Korea (Grant No. 2017R1A2B4005087 and Grant No. 2019R1A2C1007323, 2020R1A2 B5B01002278).

\section{Authors' contributions}

YG performed cell culture and animal work, and collected data. JO designed experimental details, performed data analysis and interpretation, and wrote the manuscript. JSK conceived the experimental ideas, and finally approved the manuscript. All authors read and approved the final manuscript.

\section{Availability of data and materials}

The authors declare that all data supporting the findings of this study are available in the article and can be provided by the corresponding author on reasonable request.

\section{Competing Interests}

The authors declare no competing interests.

\section{Author details}

1 School of Food Science and Biotechnology, Kyungpook National University, Daegu 41566, Republic of Korea. ${ }^{2}$ Institute of Agricultural Science and Technology, Kyungpook National University, Daegu 41566, Republic of Korea. 
Received: 11 November 2020 Accepted: 4 December 2020

Published online: 12 December 2020

\section{References}

1. Fahey JW, Talalay P (1999) Antioxidant functions of sulforaphane: a potent inducer of Phase II detoxication enzymes. Food Chem Toxicol 37:973-979

2. Thimmulappa RK, Mai KH, Srisuma S, Kensler TW, Yamamoto M, Biswal S (2002) Identification of Nrf2-regulated genes induced by the chemopreventive agent sulforaphane by oligonucleotide microarray. Cancer Res 62:5196-5203

3. Poss KD, Tonegawa S (1997) Reduced stress defense in heme oxygenase 1-deficient cells. Proc Natl Acad Sci USA 94:10925-10930

4. Na HK, Surh YJ (2014) Oncogenic potential of Nrf2 and its principal target protein heme oxygenase-1. Free Radic Biol Med 67:353-365

5. Ma Q (2013) Role of nrf2 in oxidative stress and toxicity. Annu Rev Pharmacol Toxicol 53:401-426

6. Kubo E, Chhunchha B, Singh P, Sasaki H, Singh DP (2017) Sulforaphane reactivates cellular antioxidant defense by inducing Nrf2/ARE/Prdx6 activity during aging and oxidative stress. Sci Rep 7:14130

7. Kang KW, Lee SJ, Kim SG (2005) Molecular mechanism of nrf2 activation by oxidative stress. Antioxid Redox Signal 7:1664-1673

8. Menegon S, Columbano A, Giordano S (2016) The dual roles of NRF2 in cancer. Trends Mol Med 22:578-593

9. Sporn MB, Liby KT (2012) NRF2 and cancer: the good, the bad and the importance of context. Nat Rev Cancer 12:564-571

10. Gonzalez-Donquiles C, Alonso-Molero J, Fernandez-Villa T, VilorioMarques L, Molina AJ, Martin V (2017) The NRF2 transcription factor plays a dual role in colorectal cancer: a systematic review. PLOS ONE 12:e0177549

11. Lane DP (1992) Cancer. p53, guardian of the genome. Nature 358:15-16

12. Vousden $\mathrm{KH}$, Prives C (2009) Blinded by the light: the growing complexity of p53. Cell 137:413-431

13. Marine JC, Lozano G (2010) Mdm2-mediated ubiquitylation: p53 and beyond. Cell Death Differ 17:93-102

14. Rotblat B, Melino G, Knight RA (2012) NRF2 and p53: Januses in cancer? Oncotarget 3:1272-1283

15. Woo Y, Oh J, Kim JS (2017) Suppression of Nrf2 activity by chestnut leaf extract increases chemosensitivity of breast cancer stem cells to paclitaxel. Nutrients 9:760

16. Seo H, Oh J, Hahn D, Kwon CS, Lee JS, Kim JS (2017) Protective effect of glyceollins in a mouse model of dextran sulfate sodium-induced colitis. J Med Food 20:1055-1062

17. Gorrini C, Harris IS, Mak TW (2013) Modulation of oxidative stress as an anticancer strategy. Nat Rev Drug Discov 12:931-947

18. Liou GY, Storz P (2010) Reactive oxygen species in cancer. Free Radic Res 44:479-496

19. Moon EJ, Giaccia A (2015) Dual roles of NRF2 in tumor prevention and progression: possible implications in cancer treatment. Free Radic Bio Med 79:292-299

20. Ryoo IG, Lee SH, Kwak MK (2016) Redox modulating NRF2: a potential mediator of cancer stem cell resistance. Oxid Med Cell Longev 2016:2428153

21. Wang XJ, Sun Z, Villeneuve NF, Zhang S, Zhao F, Li Y, Chen W, Yi X, Zheng W, Wondrak GT et al (2008) Nrf2 enhances resistance of cancer cells to chemotherapeutic drugs, the dark side of Nrf2. Carcinogenesis 29:1235-1243

22. Kensler TW, Wakabayashi N, Biswal S (2007) Cell survival responses to environmental stresses via the Keap1-Nrf2-ARE pathway. Annu Rev Pharmacol Toxicol 47:89-116

23. Surh YJ, Kundu JK, Na HK (2008) Nrf2 as a master redox switch in turning on the cellular signaling involved in the induction of cytoprotective genes by some chemopreventive phytochemicals. Planta Med 74:1526-1539

24. Kensler TW, Egner PA, Agyeman AS, Visvanathan K, Groopman JD, Chen JG, Chen TY, Fahey JW, Talalay P (2013) Keap1-nrf2 signaling: a target for cancer prevention by sulforaphane. Top Curr Chem 329:163-177

25. Faraonio R, Vergara P, Di Marzo D, Pierantoni MG, Napolitano M, Russo T, Cimino F (2006) p53 suppresses the Nrf2-dependent transcription of antioxidant response genes. J Biol Chem 281:39776-39784
26. Chen W, Sun Z, Wang XJ, Jiang T, Huang Z, Fang D, Zhang DD (2009) Direct interaction between Nrf2 and p21(Cip1/WAF1) upregulates the Nrf2-mediated antioxidant response. Mol Cell 34:663-673

27. Komatsu M, Kurokawa H, Waguri S, Taguchi K, Kobayashi A, Ichimura Y, Sou YS, Ueno I, Sakamoto A, Tong Kl et al (2010) The selective autophagy substrate $\mathrm{p} 62$ activates the stress responsive transcription factor Nrf2 through inactivation of Keap1. Nat Cell Biol 12:213-223

28. Chen WM, Jiang T, Wang HH, Tao SS, Lau A, Fang DY, Zhang DD (2012) Does Nrf2 contribute to p53-mediated control of cell survival and death? Antioxid Redox Signal 17:1670-1675

29. Luo Q, Beaver JM, Liu Y, Zhang Z (2017) Dynamics of p53: a master decider of cell fate. Genes (Basel) 8:66

30. Martindale JL, Holbrook NJ (2002) Cellular response to oxidative stress: signaling for suicide and survival. J Cell Physiol 192:1-15

31. Bensaad K, Cheung EC, Vousden KH (2009) Modulation of intracellular ROS levels by TIGAR controls autophagy. EMBO J 28:3015-3026

32. Crighton D, Wilkinson S, O'Prey J, Syed N, Smith P, Harrison PR, Gasco M, Garrone O, Crook T, Ryan KM (2006) DRAM, a p53-induced modulator of autophagy, is critical for apoptosis. Cell 126:121-134

33. Lee J, Giordano S, Zhang J (2012) Autophagy, mitochondria and oxidative stress: cross-talk and redox signalling. Biochem J 441:523-540

34. Moulder DE, Hatoum D, Tay E, Lin YG, McGowan EM (2018) The roles of p53 in mitochondrial dynamics and cancer metabolism: the pendulum between survival and death in breast cancer? Cancers (Basel) 10:189

35. Bourdon A, Minai L, Serre V, Jais JP, Sarzi E, Aubert S, Chretien D, de Lonlay $\mathrm{P}$, Paquis-Flucklinger $\mathrm{V}$, Arakawa $\mathrm{H}$ et al (2007) Mutation of RRM2B, encoding p53-controlled ribonucleotide reductase (p53R2), causes severe mitochondrial DNA depletion. Nat Genet 39:776-780

36. Lebedeva MA, Eaton JS, Shadel GS (2009) Loss of p53 causes mitochondrial DNA depletion and altered mitochondrial reactive oxygen species homeostasis. Biochim Biophys Acta 1787:328-334

37. Dinkova-Kostova AT, Abramov AY (2015) The emerging role of Nrf2 in mitochondrial function. Free Radic Biol Med 88:179-188

38. Piantadosi CA, Carraway MS, Babiker A, Suliman HB (2008) Heme oxygenase-1 regulates cardiac mitochondrial biogenesis via Nrf2mediated transcriptional control of nuclear respiratory factor-1. Circ Res 103:1232-1240

39. Strom J, Xu B, Tian X, Chen QM (2016) Nrf2 protects mitochondrial decay by oxidative stress. FASEB J 30:66-80

40. Espinosa-Diez C, Miguel V, Mennerich D, Kietzmann T, Sanchez-Perez P, Cadenas S, Lamas S (2015) Antioxidant responses and cellular adjustments to oxidative stress. Redox Biol 6:183-197

41. Simabuco FM, Morale MG, Pavan ICB, Morelli AP, Silva FR, Tamura RE (2018) p53 and metabolism: from mechanism to therapeutics. Oncotarget 9:23780-23823

42. Boland ML, Chourasia AH, Macleod KF (2013) Mitochondrial dysfunction in cancer. Front Oncol 3:292

43. Vyas S, Zaganjor E, Haigis MC (2016) Mitochondria and cancer. Cell 166:555-566

44. Tian M, Xu XY, Hu H, Liu Y, Pan SY (2017) Optimisation of enzymatic production of sulforaphane in broccoli sprouts and their total antioxidant activity at different growth and storage days. J Food Sci Tech Mys 54:209-218

45. Kim M, Lee K, Kim J, Sok D (1997) Determination of sulforaphane in cruciferous vegetables by SIM. Korean J Food Sci Technol 29:882-887

46. Reagan-Shaw S, Nihal M, Ahmad N (2008) Dose translation from animal to human studies revisited. Faseb J 22:659-661

47. Lekcharoenkul P, Tanongkankit Y, Chiewchan N, Devahastin S (2014) Enhancement of sulforaphane content in cabbage outer leaves using hybrid drying technique and stepwise change of drying temperature. J Food Eng 122:56-61

\section{Publisher's Note}

Springer Nature remains neutral with regard to jurisdictional claims in published maps and institutional affiliations. 